\title{
Forced apart: a microtubule-based mechanism for equidistant positioning of multiple nuclei in single cells
}

\author{
Juliane Teapal $^{1}$, Leander J. Schuitman ${ }^{1,2}$, Bela M. Mulder ${ }^{1,2,3, a}{ }_{\mathbb{D}}$, Marcel E. Janson ${ }^{1}$ \\ ${ }^{1}$ Laboratory of Cell Biology, Wageningen University, Droevendaalsesteeg 1, 6708 PB Wageningen, \\ The Netherlands \\ 2 Living Matter Department, Institute AMOLF, Science Park 104, 1098 XG Amsterdam, The Netherlands \\ 3 Departments of Theoretical Physics and Biology, Utrecht University, Padualaan 8, $3584 \mathrm{CH}$ Utrecht, The \\ Netherlands
}

Received: 19 December 2020 / Accepted: 6 July 2021

(C) The Author(s) 2021

\begin{abstract}
Cells can position multiple copies of components like carboxysomes, nucleoids, and nuclei at regular intervals. By controlling positions, cells, for example, ensure equal partitioning of organelles over daughter cells and, in the case of nuclei, control cell sizes during cellularization. Mechanisms that generate regular patterns are as yet poorly understood. We used fission yeast cell cycle mutants to investigate the dispersion of multiple nuclei by microtubule-generated forces in single cells. After removing internuclear attractive forces by microtubule-based molecular motors, we observed the establishment of regular patterns of nuclei. Based on live-cell imaging, we hypothesized that microtubule growth within internuclear spaces pushes neighbouring nuclei apart. In the proposed mechanism, which was validated by stochastic simulations, the repulsive force weakens with increasing separation because stochastic shortening events limit the extent over which microtubules generate forces. Our results, therefore, demonstrate how cells can exploit the dynamics of microtubule growth for the equidistant positioning of organelles.
\end{abstract}

\section{Introduction}

Cells are highly compartmentalized and the correct positioning of cellular components is crucial for many aspects of cellular functioning. During specific cellular stages, it is, moreover, required that multiple copies of components become arranged at regular intervals. Examples are found in both prokaryotic and eukaryotic cells: the spacing of microcompartments and chromosomes in prokaryotes ensures that daughter cells inherit an equal number of copies during cell division $[1,2]$, ordered arrays of basal bodies and adhered cilia in multiciliated cells [3] generate efficient transport of extracellular fluid, equidistant nuclei in multinucleated embryonic cells [4] control cell sizes during cellularization, and nuclear positioning is thought to be required for the proper functioning of multinucleated muscle cells [5]. In eukaryotic cells, the cellular cytoskeleton is generally implicated in the positioning of organelles; how-

\footnotetext{
a e-mail: mulder@amolf.nl (corresponding author)
} 
ever, the establishment of equidistant patterns by cytoskeletal forces is seldom understood at a mechanistic level.

To improve our understanding of organelle spacing mechanisms, we studied nuclear patterns in fission yeast (Schizosaccharomyces pombe) cells with multiple nuclei. Normally, wild-type fission yeast cells have a single nucleus during interphase. From this nucleus, plus ends of microtubules grow away and generate pushing forces in contact with cell ends. Collectively these forces push the nucleus towards the centre of the longitudinal cells [6]. Fission yeast cells harbour two nuclei after cell fusion events. After cell fusion, two nuclei will congress and fuse in a process called karyogamy. For this, nuclear-associated microtubules form an internuclear bundle that contracts by minus-end-directed motor proteins dynein and kinesin-14 [7, 8]. Generation of internuclear attractive forces by minus-end-directed motors was, furthermore, demonstrated during cell division when duplicated nuclei momentarily share a single cell before cytokinesis divides the cell in two. These nuclei will unwantedly congress in cells that fail to downregulate the activity of the minus-end-directed kinesin-14 motor protein klp2p [9].

Fission yeast cells with more than two nuclei can be experimentally obtained using mutations in cell cycle genes that cause cells to undergo multiple rounds of nuclear division without completing cytokinesis. We observed that these mutants can form equidistant nuclear patterns in the absence of motor-driven internuclear attractive forces. Live-cell imaging suggested that microtubules generated repulsive forces by growing from one nucleus towards a neighbouring nucleus. The frequency of these interactions is expected to decrease with internuclear distance because the onset of microtubule shortening, termed a catastrophe, limits the length over which microtubules can generate force. Stochastic switching between microtubule growth and shrinkage (dynamic instability) thus ensures that the net repulsion force between nuclei is distance-dependent, a prerequisite for the formation of equidistant patterns.

\section{Results and discussion}

To investigate the positioning of nuclei in multinucleated fission yeast mutants, we grew cytokinesis defective $c d c 11$ cells expressing a fluorescent marker for the nuclear envelope $[6,10]$. After two rounds of nuclear division without subsequent cytokinesis, $c d c 11$ cells contained four nuclei that clustered together near the cell centre (Fig. 1a and video 1). To reduce internuclear attractive forces, we imaged cells that additionally lacked the motor protein klp2p, which slides antiparallel microtubules apart in interphase cells [11, 12]. Nuclei in these cells did not cluster anymore but were regularly distributed along the cell length (Fig. 1a and video 2). Some degree of nuclear patterning is a priori expected in the absence of attractive forces because nuclei cannot overlap, which in effect generates a repulsive force between them [13]. The distribution of nuclear positions and consequently the distribution of internuclear distances were, however, narrower than expected for random positioning in the presence of overlap constraints alone (Fig. 1b, Fig. S1, A and B). The mean internuclear distance was proportional to the cell length for cells between 25 and $50 \mu \mathrm{m}$ long, whereas its coefficient of variation was approximately constant or potentially decreasing (Fig. S1C). Removal of internuclear attractive forces thus exposed a hidden positioning mechanism that was adaptive to cell length.

To gain more insight into the forces that drive the formation of equidistant patterns, we observed the dynamics of nuclei after displacing them by centrifugal forces towards one cell end [14]. Nuclei in $c d c 11$ cells with klp2p moved as a single cluster back towards the 


\section{A} cdc11

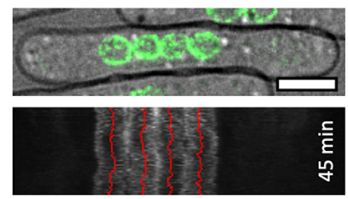

C

cdc11
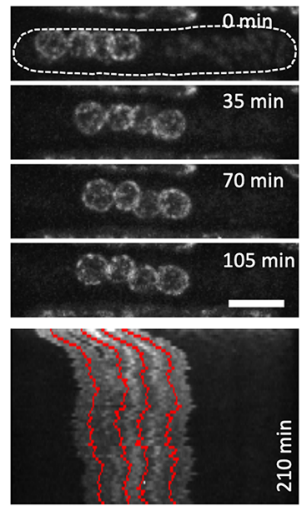

cdc11 klp2s

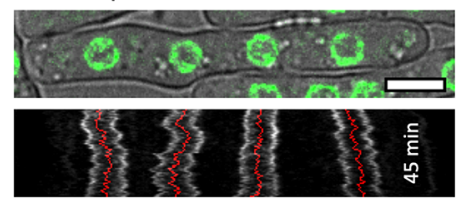

cdc11 klp2 $\Delta$
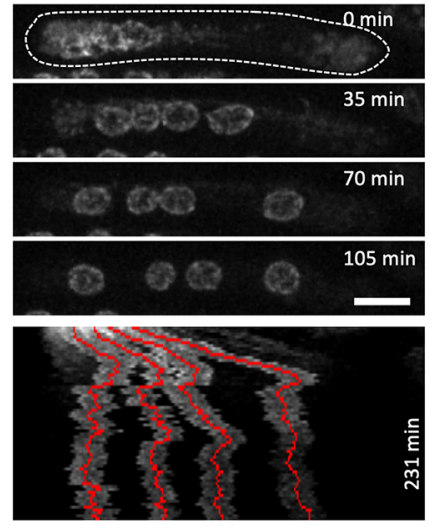

B

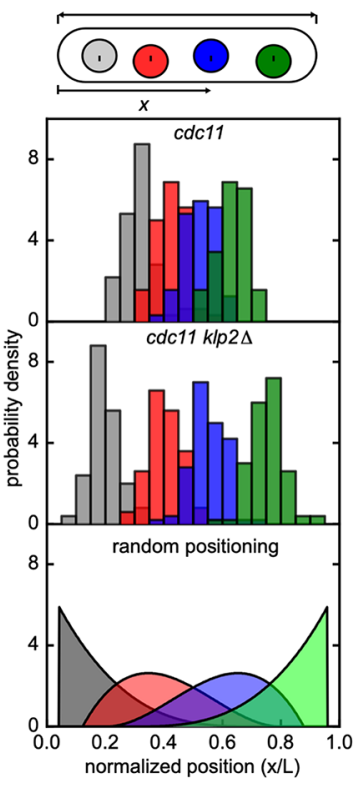

Fig. 1 Equidistant positioning of nuclei in the absence of klp2p. A Confocal images, overlaid on DIC images, of tetranucleated $c d c 11$ and $c d c 11 \mathrm{klp} 2 \Delta$ cells expressing the nuclear envelope marker nup107-GFP. The kymographs below are an xt-scan of the fluorescence intensity in which the red lines indicate the midpoints of nuclei. B Distribution of nuclear midpoints positions in tetranucelated $c d c 11$ ( $n=64$ cells) and $c d c 11 \mathrm{klp} 2 \Delta$ cells $(n=100$ cells). The positions are normalized to the length of individual cells. The bottom panel shows the theoretical distribution for random positioning of non-overlapping nuclei (Eq. 6). C Upper panel: Confocal images showing nuclear motion after cell centrifugation in tetranucleated $c d c 11$ and $c d c 11 \mathrm{klp} 2 \Delta$ cells. Dotted line indicates the cell outline. Cell autofluorescence decreased the visibility of nuclei, particularly at the start of imaging which commenced approximately 3.5 minutes after centrifugation. Bottom panel: kymograph of nuclear dynamics. Scale bars: $5 \mu \mathrm{m}$

cell centre (Fig. 1c and video 3). In contrast, nuclei in $c d c 11$ cells lacking klp2p dispersed and regained their steady-state positions within approximately $2.5 \mathrm{~h}$ (Fig. 1c and video 4). The re-establishment of nuclear patterns occurred during interphase, before nuclei divided in the next mitosis, and could be prevented by the addition of the microtubule-depolymerizing drug MBC (Fig. S1D). Interphase microtubules thus appeared to be required for equidistant positioning.

Microtubules in fission yeast cells are arranged into three to five interphase microtubule assemblies (IMAs) [6, 11, 12, 15, 16]. Each assembly is dynamic and consists out of several microtubules arranged in a linear manner with microtubule growth primarily directed away from the nucleus. A central region of microtubule overlap in each IMA is in contact with the nuclear envelope (NE) and one IMA is, moreover, connected to the NE-embedded spindle pole body (SPB), the yeast analogue of a centrosome. To characterize the behaviour of microtubules in multinucleated cells, we imaged microtubule dynamics in $c d c 11 \mathrm{klp} 2 \Delta$ cells expressing different fluorescent markers for the NE and SPB. For simplicity, we imaged microtubules in cells with two instead of four nuclei. Microtubules were oriented primarily along the cell's long axis and were both present in between the nuclei and between the nuclei and cell ends (Fig. 2a and video 5). SPBs moved back and forth over the NEs like in wild-type 
cells, indicating that microtubule pushing forces are transmitted to the nuclei (Fig. 2a; and video 6) [6]. Microtubules originating from neighbouring nuclei were occasionally observed to form internuclear bundles that in some cases bridged two SPBs (Fig. 2b). In wild-type cells, these events caused SPBs and nuclei to move together, very much reminiscent to cytokinesis in cells with active klp2p [9]. In contrast, in cells lacking klp2p, nuclei stayed apart upon the formation of internuclear bundles, demonstrating that removal of klp2p eliminated internuclear forces through bundle formation. This is in agreement with earlier work that showed that inter-microtubule sliding within IMAs ceases in the absence of klp2p $[11,12]$. Occasionally, a microtubule originating from one nucleus impinged onto the surface of a neighbouring nucleus and then grew along it or underwent a catastrophe (Fig. $2 \mathrm{c}$ and video 7). We hypothesized that these events generated repulsive forces between nuclei that could space nuclei apart in the absence of klp2p-dependent clustering forces. We summarized the observed interactions of microtubules in multinucleated cells in Fig. $2 \mathrm{~d}$.

Inspired by our experimental results, we developed a 1-dimensional stochastic simulation to test if microtubule pushing forces can explain the dispersion of nuclei. In this model, microtubule minus-ends were attached to nuclei that moved when growing plus-ends impinged onto neighbouring nuclei or cell ends. Forces as well as rates of microtubule nucleation and dynamic instability were based on experimental data (Table 2). We employed a microtubule force-production model that takes into account that forces on microtubule ends lower their growth rate as well as increase their catastrophe probability. Different from previous models, the rate at which forces build up after microtubules impact upon cell walls or nuclei was governed by a single effective compressional modulus (Eq. 11). This phenomenological parameter was chosen such that force-induced catastrophes limited the average microtubule interaction time with cell ends to the experimentally observed mean residence time of $90 \mathrm{~s}$ previously measured in mononucleated cells [6].

In support of our proposed mechanism, the simulated nuclei in tetranucleated cells attained equidistant patterns within the confining space (Fig. 3a). Both the distribution of nuclear midpoints and the timescale of pattern re-establishment after perturbation were in good agreement with experimental data (Fig. $3 \mathrm{~b}$, c for passing probability $=0$ ). The outer nuclei were, however, positioned closer to the cell ends than observed in cells, suggesting that force generation in between nuclei was overestimated relative to force generation in between outer nuclei and cell ends (Fig. $3 \mathrm{~b}$ for passing probability $=0$ ). To resolve this discrepancy, we introduced a nuclear passing probability based on the possibility that microtubules can grow past a neighbouring nucleus but cannot pass a cell end. A probability of $72.5 \%$ for a microtubule to pass nuclei corrected the positions of outer nuclei while maintaining good agreement for repositioning timescales (Fig. 3b, c). The introduction of nuclear passing also recapitulated a small observed variation in internuclear spacing, whereby the distance between the two inner nuclei was on average the shortest (Fig. S1B; and Fig. 4a). Without nuclear passing, the available space between the two outer nuclei was divided in three equal parts by the two inner nuclei, as expected since interactions between all neighbouring nuclei are equal (data not shown). However, with the introduction of nuclear passing, microtubules from the inner nuclei occasionally pushed against cell ends, generating an additional inwarddirected force, explaining the slightly shorter distance between inner nuclei. In conclusion, the dynamics and magnitude of microtubule polymerization forces can quantitatively explain the near equidistant patterning of nuclei in yeast cells if the differential efficiency of force generation against cell walls and nuclei is taken into account.

The observed variance of the internuclear distances in our simulations can be attributed to temporal fluctuations in pushing forces due to a finite number of contributing microtubules. To further investigate conditions for efficient positioning, we changed the average num- 
A

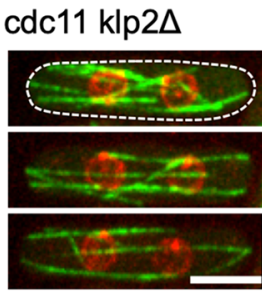

cdc11 klp2 $\Delta$

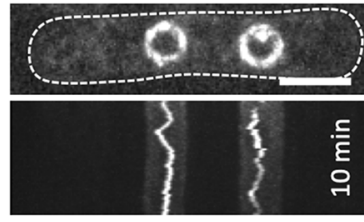

B

cdc11
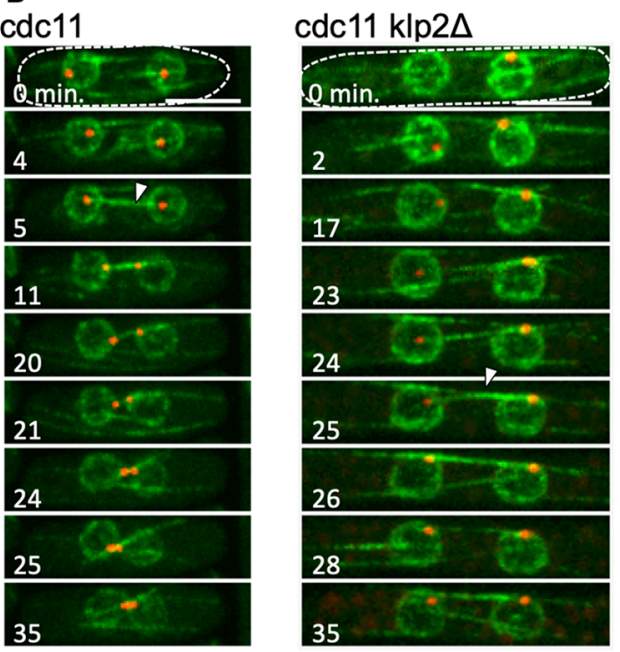

D

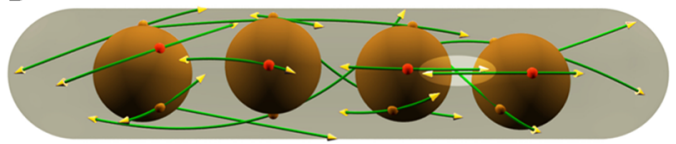

C cdc11 klp2 $\Delta$
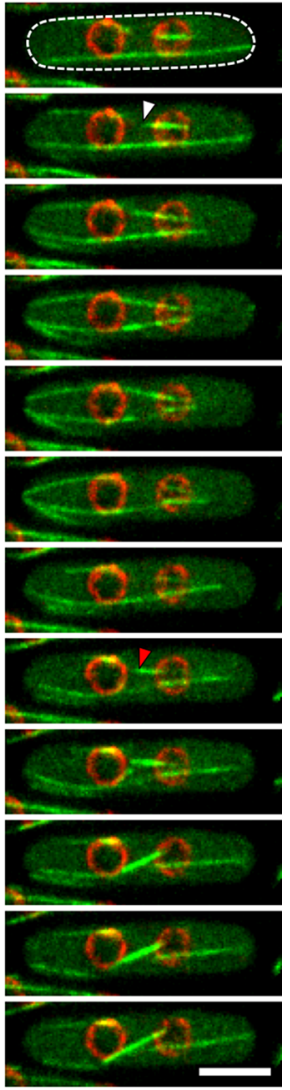

Fig. 2 Force generation by bidirectional microtubule growth in internuclear spaces. A Left: Confocal images of a binucleated $c d c 11 \mathrm{klp} 2 \Delta$ cell expressing GFP-tubulin, the spindle pole body (SPB) marker sid4-RFP, and the nuclear envelope (NE) marker cut11-3mRFP. Displayed images are taken 6 minutes apart and show the full dept of the cell. Right: same cells but only showing the SPB and NE signals. The kymograph depicts SPB movement over the NE. B Confocal images of binucleated $c d c 11$ and $c d c 11 k l p 2 \Delta$ cells expressing GFP-atb2, nup107-GFP (NE) and Sid4-RFP (SPB). Images were captured directly after the disassembly of the mitotic spindles that pushed the two nuclei apart in the preceding mitosis, so before nuclear clustering occurred in $c d c 11$ cells. Displayed timepoints were selected to show the formation and dynamics of internuclear microtubule bundles that connect SPBs; events are indicated by arrowheads. C Impingement of growing microtubule ends on nuclear envelopes visualized in $c d c 11 \mathrm{klp} 2 \Delta$ cells expressing GFP-atb2, sid4-RFP (SPB) and cut11-3mRFP (NE). Images are confocal projections of 3 confocal planes and are taken $25 \mathrm{~s}$ apart. The first microtubule (white arrowhead) grows leftwards towards a neighbouring nucleus and has a catastrophe after contacting it. The second microtubule (red arrowhead) moves over the neighbouring nuclei and then continues to grow past it. d Graphical representation of proposed microtubule interactions in tetranucleated cells. Multiple microtubules with opposite polarity, two are shown for simplicity, are arranged into microtubule assemblies (IMAs). The central region of each IMA is connected to a nucleus. One IMA per nucleus is also connected to a SPB (red sphere). Growing microtubule plus-ends (yellow cones) generate pushing forces against cell walls and nuclei. Occasionally, internuclear bundles are established by microtubules growing from two neighbouring nuclei (highlighted elliptical area). These bundles contract in the presence of klp2p. Note that the temporal establishment of an internuclear bundle in a 3-dimensional cell does not necessarily impede the relative movement of nuclei because microtubules can pivot at their nuclear attachment sites. Scale bars: $5 \mu \mathrm{m}$ 
A
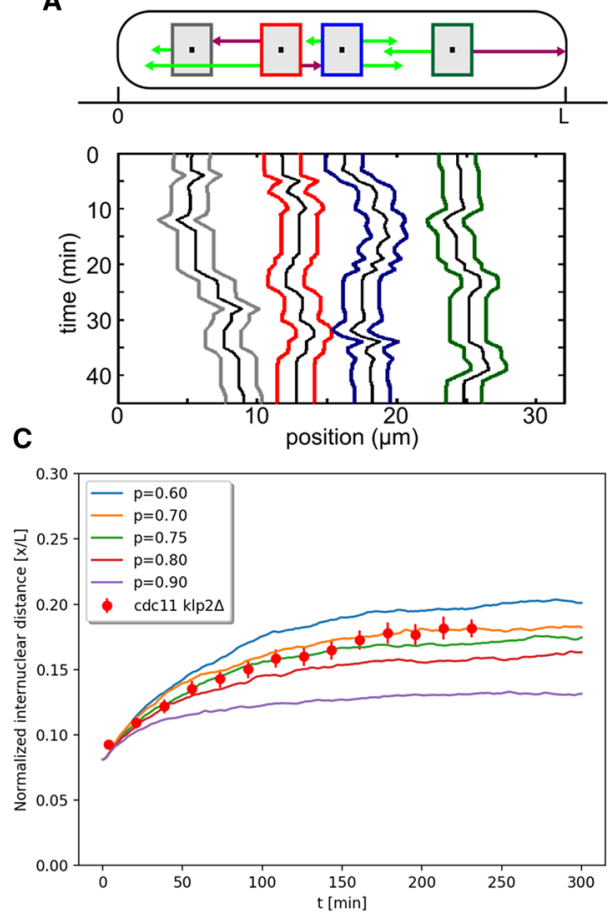

B
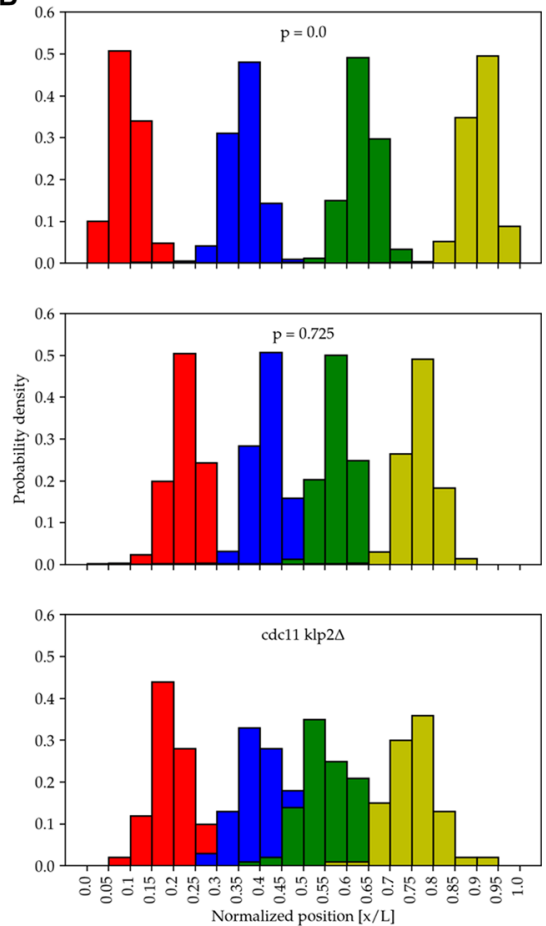

Fig. 3 1D stochastic simulation supports nuclear positioning by microtubule pushing forces. A Upper panel: Graphic representation of the simulation. Microtubules, represented by arrows, grow away from nuclei and push (red arrows) against cell ends and neighbouring nuclei. Microtubules can pass neighbouring nuclei without interaction with a probability $p$. Bottom panel: Time evolution of simulated nuclear positions visualized as a kymograph. B Distribution of nuclear midpoint positions in simulations with four nuclei for nuclear passing probabilities of $0 \%$ (upper panel) and $72.5 \%$ (middle panel) $(n=200$ simulations for each condition). The lower panel repeats the experimental data of cdc1 $1 \mathrm{klp} 2 \Delta$ cells from Fig. $1 \mathrm{~b}$ for comparison. C Simulated time evolution of the mean internuclear distance, measured between midpoints and scaled with cell length (averaged over $n=200$ simulations). Nuclei were close packed at one side of the simulated cell at $t=0$. Red points show experimental data for cdc $11 \mathrm{klp} 2 \Delta$ cells after cell centrifugation $(n=109$ cells $)$

ber of microtubules per nucleus (Fig. 4a). As expected, the fluctuations in internuclear distances decreased monotonically as the contributions of single microtubules averaged out with increasing numbers of interactors [17]. Largest relative fluctuations were observed between the two inner nuclei in both the simulations and the experiments (Fig. S1B and Fig. 4a). This can be understood, because when a nucleus is ranked further away from the cell wall, it loses its relation to the cell wall, which acts like a fixed reference point. A previous 1-dimensional model on microtubule-based positioning in confining geometries suggested that positioning may slow down in cells if simultaneous pushing interactions on both sides of an object block motion [18]. Such a geometry frequently occurs in our simulations at increased microtubule densities. However, the compliances in our model allowed for nuclear motion even if microtubules simultaneously generated forces on both sides. The elastic restoring force, therefore, scaled with the number of microtubules and repositioning sped up consequently in the simulations (Fig. 4a).

Earlier, it was proposed that equidistant spacing of nuclei in multinucleated muscle cells requires repulsive forces that decay with increasing internuclear distance [5, 19-21]. 
A
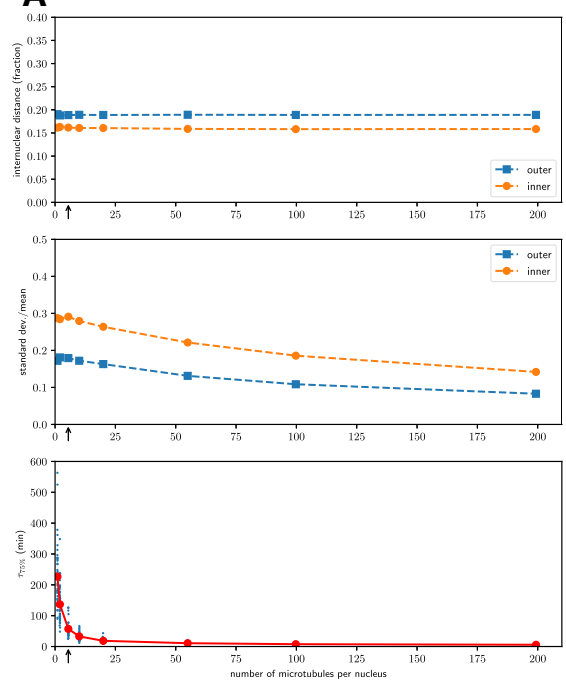

B
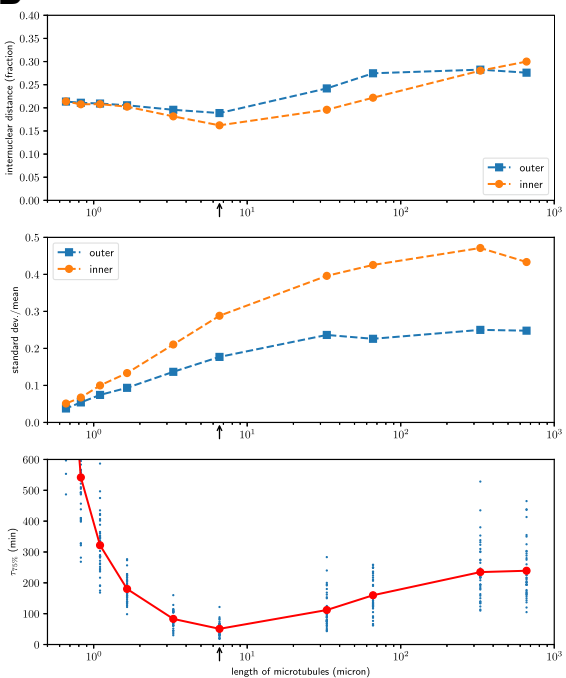

Fig. 4 Parameter sweeps 1D stochastic simulations. A Simulation outcomes for different values of the microtubule nucleation rate, $r_{n}$. The resulting average number of microtubules adhered per nucleus, 5.5 for the default value of $r_{n}$ in Table 2 (arrow), is plotted along the horizontal axis. All other parameters are as listed in Table 2, except the passing probability, which is set to the best-fit value of $p_{\text {pass }}=0.725$. Upper panel shows the mean internuclear distance, discriminating between inner and outer internuclear distances, and normalized to the cell length. Middle panel shows the corresponding coefficient of variation $(\sigma / \mu)$. See caption of Fig. $\mathrm{S} 1 \mathrm{~B}$ for experimental values. Lower panel shows the average timescale for nuclear redistribution, $\tau_{75} \%$. This timescale was calculated for each simulated cell as the time in which the average of the three internuclear distances first rose from its closed-packed value $(0 \%)$ to $75 \%$ of the steady-state value (100\%). B Same as in (B) but for variation of the unconstrained catastrophe rate $r_{c}{ }^{(0)}$. The horizontal axis indicates the characteristic length scale, $v_{\mathrm{g}}{ }^{(0)} / r_{\mathrm{c}}{ }^{(0)}$, which would be the mean of the microtubule length distribution in case of unconstrained growth; $6.6 \mu \mathrm{m}$ for the default values in Table 2 (arrow). Note that the catastrophe rate in the simulation was only changed for microtubules growing freely in the cytoplasm. The catastrophe rate for modelling catastrophes of force-generating microtubules was left unchanged with the goal of keeping the duration and leverage of individual microtubule impacts similar while changing the length distribution of microtubules. In all cases, averages are shown over $\mathrm{n}=40$ independent simulations, while for $\tau_{75 \%}$, the full scatter over these replicates is also shown

Microtubule-generated forces in our 1-dimensional simulations of yeast cells fulfil this requirement for two reasons: Firstly, unconstrained catastrophes in the cytoplasm limit the extend by which microtubules can grow away from nuclei. And, secondly, as distance increases, microtubules spend relatively more time in non-force generating states that include the growth time towards a site of force generation and subsequently the shrinkage time after a barrier-induced catastrophe [17]. To investigate how unconstrained catastrophes contribute to the positioning of nuclei, we varied the catastrophe rate for unconstrained microtubules in our simulations while leaving the dynamics of barrier-induced catastrophes unaltered (Fig. 4b). Fastest positioning was achieved when the characteristic length of microtubules, $L^{*}$, equal to the ratio of growth velocity and unconstrained catastrophe rate was similar to the internuclear distance. In fission yeast, $L^{*}$ is $6.6 \mu \mathrm{m}$ and is close to this optimal value (Table 2). Positioning in our simulations slowed down significantly for $L^{*}$-values larger than the internuclear distance, a parameter regime in which unconstrained catastrophes are rare. Slower positioning was also observed for $L^{*}$-values smaller than the internuclear distance because frequent unconstrained catastrophes prevented the majority of microtubules 
from reaching their targets. On the other hand, fluctuations in internuclear distances were minimalized for these small $L^{*}$-values, similarly to what was previously described for the microtubule-based centring of a single object in a confining geometry [18]. In essence, the latter can be explained by looking at a nucleus that is slightly displaced from its equilibrium position. The probability that a microtubule generates a pushing event towards the equilibrium position without undergoing a premature unconstrained catastrophe is larger than the probability of pushing in the opposite direction for which a longer distance needs to be spanned. This asymmetry in probabilities increases with decreasing $L^{*}$, in effect preventing large fluctuations away from the equilibrium position. Lastly, for large $L^{*}$-values, the internuclear distances increased at the expense of the space between outer nuclei and cell ends. This indicated that in the absence of unconstrained catastrophes, the force that pushes nuclei inwards towards the cell centre decreases in strength relative to the force that pushes nuclei apart. In conclusion, our simulations demonstrate that barrier-induced catastrophes are sufficient for achieving equidistant positioning of nuclei, but unconstrained catastrophes, especially at the rate observed in yeast, are required to reduce fluctuations in internuclear distances and to speed up nuclear repositioning to the levels observed in tetranucleated cells (Fig. S1B and Fig. 3c).

From our experiments and simulations, we concluded that microtubule pushing forces can explain the dynamics and accuracy of equidistant nuclear positioning in multinucleated fission yeast. Since microtubules generally radiate away from cellular nuclei, it is reasonable to assume that pushing forces contribute to nuclear repulsion in a diverse range of cell types that harbour multiple nuclei. These include filamentous fungi, myotubes, plant cells, algae, and embryos [5, 22-25]. Numerous studies demonstrated, however, that microtubule-based motor proteins generate additional forces on nuclei in these cells. In cells of the filamentous fungi Ashbya gossypii, interactions between long microtubules and cortical dynein power long-ranged nuclear motility [26, 27]. Shorter microtubules, which are also present [28], could, however, generate short-ranged nuclear repulsion like the microtubules in fission yeast. In myotubes, motors are thought to move nuclei in two ways: by sliding microtubules from two neighbouring nuclei apart [5], and, when attached to nuclear envelopes, by pulling nuclei directly along microtubules from neighbouring nuclei [29]. Microtubules-based pushing forces may, therefore, generate a basal level of control, but cells in which equidistant positioning of nuclei serves an important biological function have evolved additional motor-based mechanisms to control internuclear forces.

In summary, aided by the simple architecture and amenability of the fission yeast cell, we uncovered a mechanism by which microtubules can position cellular objects at equidistant intervals. The mechanism was verified on an extensive dataset of tetranucleated cells but can be further tested on cells with two or eight nuclei which can be obtained by changing growth times of $c d c 11$ cells under restrictive conditions. Moreover, the forceproduction relationships can be adapted to investigate which aspects of microtubule dynamics under load are most important to achieve efficient nuclear positioning in our model. Interestingly, the proposed mechanism does not involve the classical elements of reactiondiffusion models that set length scales in diverse biological processes, including the equidistant distribution of plasmids in prokaryotes [30,31]. There are, however, strong mechanistic similarities in the sense that a signal (microtubule) is activated locally (nucleation near the nucleus), while it is deactivated elsewhere on a certain time scale (catastrophe rate) in which it has bridged a characteristic length scale. It will be interesting to learn how similar scenarios are played out to control organelle spacing in other cellular systems. 


\section{Materials and methods}

\subsection{Generation of yeast strains}

Standard media and genetic methods were used as described in the Nurse Lab Handbook. Strains used are listed in Table 1. All strains were confirmed by PCR.

\subsection{Cell culture}

Before imaging, temperature-sensitive $c d c 11$ cells were grown in liquid YE5S media at a permissive temperature of $25{ }^{\circ} \mathrm{C}$ overnight. The temperature was shifted to a restrictive temperature of $36^{\circ} \mathrm{C}$ for two hours to generate binucleated cells and for five hours to generate tetranucleated cells.

\subsection{Centrifugation assay}

Displacement of nuclei by centrifugal forces was done as described [14]. Briefly, cellular microtubules were depolymerized using $25 \mu \mathrm{g} \mathrm{ml}^{-1}$ methyl-2-benzimidazole-carbamate (MBC). Directly afterwards, cells were centrifuged for $10 \mathrm{~min}$ at 14,000 $\mathrm{g}$ using an Eppendorf tabletop centrifuge. Next, the cell pellet was washed twice with growth media lacking MBC to allow for regrowth of microtubules.

\subsection{Microscopy and image analysis}

Fission yeast cells were imaged on thin EMM agar pads as described [34]. $25 \mu \mathrm{g} \mathrm{ml}^{-1} \mathrm{MBC}$ was added to the pads for nuclear repositioning assays in the absence of microtubules (Fig. S1D). Imaging was done at $25{ }^{\circ} \mathrm{C}$. To increase the length of tetranucleated cells, $12 \mathrm{mM}$ hudroxy urea (HU), which blocks DNA synthesis and consequently mitosis, was added to the agar pads (Fig. S1C). HU was also added after cell centrifugation to observe nuclear redistribution without intervening mitosis (Fig. 1c).

Table 1 Fission yeast cell lines used in the experiments

\begin{tabular}{|c|c|c|c|c|}
\hline $\mathrm{Nr}$ & Strain & & Figure & Note \\
\hline JT.44 & $h+c d c 11-123$, nup107-GFP::kanMx, ade6-M210 & ts & 1 & This study \\
\hline JT.69 & h-cdc11-123, nup107-GFP::kanMx, klp2 $\Delta::$ ura4 & ts & $1 ; \mathrm{S} 1$ & This study \\
\hline JT.140 & $\begin{array}{l}\text { cdc11-123, nup107-GFP::kanMx, sid4-RFP::kanMx, ade6-M216, } \\
\text { ura4-d18, leu1::pSV40-GFP-atb2 }\end{array}$ & ts & $2 b$ & This study \\
\hline JT.141 & $\begin{array}{l}\text { cdc11-123, klp2 } \Delta:: \text { ura4, nup 107-GFP : kanMx, } \\
\text { sid4-RFP::kanMx, ade6-M216, ura4-d18, } \\
\text { leu1::pSV40-GFP-atb2 }\end{array}$ & ts & $2 b$ & This study \\
\hline JT.162 & $\begin{array}{l}\text { cdc11-123, klp2 } \Delta:: \text { ura4, cut11-3mRFP::hph, } \\
\quad \text { leu1::pSV40-GFP-atb2, ade6-, sid4-RFP::kanMx }\end{array}$ & ts & $2 \mathrm{a}$ and $\mathrm{c}$ & This study \\
\hline PT.54 & h- nup107-GFP::kanMx & & Table S2 & Tran et al. [6] \\
\hline
\end{tabular}

Nup107-GFP, $\triangle$ klp2, sid4-RFP, GFP-atb2, and cut11-3mRFP were derived from strains constructed in, respectively [6, 8, 32, 33], and [43]. Cdc11-123 was obtained from YGRC/NBRP, Japan 
Imaging was performed using a Spinning Disc Confocal Microscope (Nikon Ti, Yokogawa CSUX1 scanner, Photometrics Evolve camera, Metamorph software, $491 \mathrm{~nm}$ and $561 \mathrm{~nm}$ laser lines, $100 \times 1.45 \mathrm{NA}$ objective). Z-stacks were acquired with a spacing of $0.5 \mu \mathrm{m}$ and maximum intensity projections are shown. For fast simultaneous acquisition of green and red spectral bands (503-552 nm and 584-900 nm), a DV2 Dual View was used (Fig. 2). Nuclear positions and cell lengths (Figs. 1b, 3c and Fig. S1) were measured manually using ImageJ software.

\subsection{Random positioning of nuclei}

We consider random positioning of nuclei with diameter $D$ into a cell of length $l$. This is equivalent to a well-known statistical mechanical model of 1D liquids called the Tonks gas. Following [35], the density of a gas of $N$ particles interacting through a pairwise potential $V(r)$, between "walls" represented by two particles fixed at $r=0$ and $r=l$, is given by

$$
\rho(r)=\frac{\sum_{k=1}^{N} L^{-1}\left[\Omega^{N-k+1}\right](l-r) L^{-1}\left[\Omega^{k}\right](r)}{L^{-1}\left[\Omega^{N+1}\right](l)}
$$

where $L^{-1}$ is the inverse Laplace transform, and

$$
\Omega(s)=L\left[e^{-\beta V}\right](s)
$$

For hard non-overlapping particles, the Boltzmann factor is simply

$$
e^{-\beta V(r)}=\theta(r-D)
$$

with $\theta(x)$ the Heaviside function. In this case, we find

$$
\Omega(s)=\int_{D}^{\infty} \mathrm{d} r e^{-s r}=\frac{e^{-s D}}{s}
$$

whence

$$
L^{-1}\left[\Omega^{n}\right](x)=\frac{(x-n D)^{n-1}}{\Gamma(n)} \theta(x-n D)
$$

For our case, we placed a fixed particle at the left boundary at $r=-\frac{1}{2} D$ and another fixed particle at the right boundary at $r=l+\frac{1}{2} D$. In this way, the edges of the flanking particles act as cell ends at $r=0$ and $r=l$, leaving the intended interval $[0, l]$ as the interior space. For $N=4$ nuclei, we thus find

$$
\begin{aligned}
\rho(r)= & \frac{\sum_{k=1}^{4} L^{-1}\left[\Omega^{4-k+1}\right](l-r) L^{-1}\left[\Omega^{k}\right]\left(r+\frac{1}{2} D\right)}{L^{-1}\left[\Omega^{5}\right]\left(l+\frac{1}{2} D\right)} \\
= & 4 \frac{\left(l-\frac{7}{2} D-r\right)^{3}}{(l-4 D)^{4}}\left(\theta\left(r-\frac{1}{2} D\right)-\theta\left(r-l-\frac{7}{2} D\right)\right) \\
& +12 \frac{\left(r-\frac{3}{2} D\right)\left(l-\frac{5}{2} D-r\right)^{2}}{(l-4 D)^{4}}\left(\theta\left(r-\frac{3}{2} D\right)-\theta\left(r-l-\frac{5}{2} D\right)\right) \\
& +12 \frac{\left(r-\frac{5}{2} D\right)^{2}\left(l-\frac{3}{2} D-r\right)}{(l-4 D)^{4}}\left(\theta\left(r-\frac{5}{2} D\right)-\theta\left(r-l-\frac{3}{2} D\right)\right)
\end{aligned}
$$


Table 2 Parameter values used in the simulation

\begin{tabular}{|c|c|c|c|}
\hline \multicolumn{2}{|l|}{ Parameter } & \multirow{2}{*}{$\begin{array}{l}\text { Value } \\
0.033 \mu \mathrm{m} \mathrm{s}^{-1}\end{array}$} & \multirow{2}{*}{$\begin{array}{l}\text { References } \\
\text { Tran et al. [6], Foethke et al. [36] }\end{array}$} \\
\hline Growth velocity & $v_{\mathrm{g}}^{(0)}$ & & \\
\hline Shrinkage velocity & $v_{\mathrm{S}}$ & $0.150 \mu \mathrm{m} \mathrm{s}^{-1}$ & Tran et al. [6], Foethke et al. [36] \\
\hline Unconstrained catastrophe rate & $r_{\mathrm{c}}^{(0)}$ & $0.005 \mathrm{~s}^{-1}$ & Tran et al. [6], Foethke et al. [36] \\
\hline Nucleation rate & $r_{n}$ & $0.0275 \mathrm{~s}^{-1}$ nucleus $^{-1}$ & $\begin{array}{l}\text { Adjusted to obtain } 1.5 \\
\text { microtubules in contact with the } \\
\text { cell wall in mononucleated cells } \\
{[37]}\end{array}$ \\
\hline Cytoplasmic viscosity & $\eta$ & $0.9 \mathrm{pNs} \mu \mathrm{m}^{-2}$ & Foethke et al. [36] \\
\hline Cell radius & $r_{\text {cell }}$ & $1.75 \mu \mathrm{m}$ & Measured \\
\hline Compressibility factor & $\kappa$ & $0.560069 \mu \mathrm{m}^{-1 \mathrm{sss}}$ & $\begin{array}{l}\text { Calculated based on the } \\
\text { microtubule residence time with } \\
\text { cell ends [6] }\end{array}$ \\
\hline Passing probability & $p_{\text {pass }}$ & Variable $(0-1)$ & \\
\hline Nuclear radius & $r_{\text {nucleus }}$ & & \\
\hline Mononucleated cells & & $1.5 \mu \mathrm{m}$ & $\begin{array}{l}\text { Measured in wild-type } \\
\text { nup107-GFP (PT54) }\end{array}$ \\
\hline Tetranucleated cells & & $1.3 \mu \mathrm{m}$ & $\begin{array}{l}\text { Measured in cdc11 klp2 } \Delta \\
\text { nup107-GFP (JT69) }\end{array}$ \\
\hline Cell length & $l_{\text {cell }}$ & & \\
\hline Mononucleated cells & & $12 \mu \mathrm{m}$ & $\begin{array}{l}\text { Measured in wild-type } \\
\text { nup107-GFP (PT54) }\end{array}$ \\
\hline Tetranucleated cells & & $32 \mu \mathrm{m}$ & $\begin{array}{l}\text { Measured in cdc11 klp2 } \Delta \\
\text { nup107-GFP (JT69) }\end{array}$ \\
\hline
\end{tabular}

$$
\begin{aligned}
& +4 \frac{\left(r-\frac{7}{2} D\right)^{3}}{(L-4 D)^{4}}\left(\theta\left(r-\frac{7}{2} D\right)-\theta\left(r-l-\frac{1}{2} D\right)\right) \\
= & \rho_{1}(r)+\rho_{2}(r)+\rho_{3}(r)+\rho_{4}(r)
\end{aligned}
$$

This is the total density, plotted in Fig. S1A, which when integrated over the interval $[0, l]$, equals the number of nuclei $(=4)$. The individual densities for the first, second, third and fourth nucleus, $\rho_{1}(r) . . \rho_{4}(r)$, are plotted in Fig. $1 \mathrm{~b}$ for parameters listed in Table 2. The nearest neighbour distance distribution (Fig. S1B) is given by

$$
\rho_{n n}(r)=4 \frac{(l-3 D-r)^{3}}{(l-4 D)^{4}}(\theta(r-D)-\theta(r-l-3 D))
$$

\subsection{D-stochastic simulation}

We simulated nuclear dispersion by microtubule pushing forces using a programme written in $\mathrm{C}++$. The simulation is one-dimensional for simplicity and speed, allowing us to focus on the core mechanism of nuclear positioning. This geometry is consistent with the elongated cylindrical geometry of the cell in which the nuclei by virtue of their size are effectively constrained to move only along the long axis of the cell. The average orientation of microtubules is along the same direction. Therefore, each nucleus has a position on an interval running from 0 to the length of the cell, and each microtubule occupies a length on this same interval. 
Since the goal of the simulation was to find the minimal conditions that are sufficient to disperse the nuclei along the cell axis, we neglected lateral interactions between microtubules. The simulation evolves the positions of the nuclei and the length of the microtubules with a fixed time step stochastic algorithm $(\Delta t=1 \mathrm{~s})$. Where possible, we validated submodules of the simulation by comparing simulation results to available and newly derived analytical predictions (see below).

\subsection{Nuclear mobility}

In fission yeast, the size of the nucleus takes up most of the cell diameter and its movement will bring about flow of viscous cytoplasm through the small remaining space between the nuclear envelope and the plasma membrane. Therefore, the viscous drag is the dominating force that counters forces generated by microtubules. We determined the nuclear mobility using the approach from Foethke et al. [36]:

$$
\mu^{t}=\frac{4 \varepsilon^{\frac{5}{2}}}{9 \pi \sqrt[2]{2} \eta_{\text {cell }} r_{\text {nucleus }}}
$$

with $\eta$ the viscosity of the cytosol and

$$
\varepsilon=\frac{r_{\text {cell }}-r_{\text {nucleus }}}{r_{\text {nucleus }}}
$$

The mobility is very sensitive to the ratio of the cell radius to the nuclear radius. Since the nuclear radius in fission yeast depends on the surrounding volume of cytoplasm [38], we estimated radii in mononucleated and tetranucleated cells for use in simulations with 1 or 4 nuclei (Table 2). With the mobility determined, we used the low Reynolds number equation of motion

$$
v_{\text {nucleus }}=\mu^{t} \times F_{\text {drag }}
$$

to solve for the nuclear velocity as a function of the forces applied, which balance the drag force $F_{\text {drag. }}$.

\subsection{Dynamic instability, target selection and force production model}

In our model, all microtubules are nucleated at the nucleus. After nucleation, the minus end of microtubules remains attached to the nucleus, whereas the plus end grows outwards. At random, a fraction, $p_{\text {pass }}$, of microtubules is chosen not to interact with any of the neighbouring nuclei, but only with the cell ends if they encounter them. Because microtubule rescues, i.e. a switch from shrinkage to growth, have not been observed away from the nucleus [6], microtubules grow until they experience a catastrophe, after which they shrink back to disappear upon reaching zero length. When not in contact with an obstacle, a microtubule has a growth speed $v_{\mathrm{g}}^{(0)}$, spontaneous catastrophe frequency $r_{\mathrm{c}}^{(0)}$ and shrinkage speed $v_{\mathrm{s}}$. When a growing microtubule contacts an obstacle, be it a nucleus or a cell wall, it can generate a force whose magnitude will depend on the "compression" it experiences as it continues to grow. In cells, compliances at the site of impact and the point of nucleation, as well as bending of the microtubule and sliding of the microtubule tip over membranes, will affect the rate at which force increases upon impact. We phenomenologically model this compression force as a single Hookean spring

$$
F=k_{\mathrm{m}}(l-L)
$$


where $l$ is the "stored length" of the microtubule and $L$ is the "compressed length", i.e. the space between the microtubule minus-end and the obstacle. We assume throughout that the compression force that microtubules bear remains smaller than the critical buckling force of microtubules. Following the experimentally observed decrease in growth velocity with force $[39,40]$, we use

$$
v_{\mathrm{g}}(F)=v_{\mathrm{g}}^{(0)} e^{-F / F_{\mathrm{s}}}
$$

with $F_{\mathrm{s}}=1.67 \mathrm{pN}$ characterizing the sensitivity to force [36]. Upon compression, the instantaneous rate of undergoing a catastrophe $r_{\mathrm{c}}(F)$ increases, which decreases the mean waiting time until a catastrophe defined by

$$
\tau_{\mathrm{c}}=\frac{1}{r_{\mathrm{c}}(F)}
$$

The mean waiting time until a catastrophe was shown to decrease approximately linearly with $v_{\mathrm{g}}$ down to $24 \mathrm{~s}$ when growth is fully stalled [41]. To aid our model, we neglected the small waiting time under stalled conditions relative to the 200 second waiting time for unconstrained growth, i.e. treat it as zero. This provides a proportional relationship between the waiting time and growth rate with a constant of proportionality equal to $1 / r_{\mathrm{c}}^{(0)} \times v_{\mathrm{g}}^{(0)}$ :

$$
\frac{1}{r_{\mathrm{c}}(F)}=\frac{1}{r_{\mathrm{c}}^{(0)} \times v_{\mathrm{g}}^{(0)}} \times v_{\mathrm{g}}(F)
$$

Given the force-velocity relation of Eq. 12, this yields

$$
r_{\mathrm{c}}(F)=r_{\mathrm{c}}^{(0)} e^{F / F_{\mathrm{s}}}
$$

which is a simplified version of the one employed in [36].

\subsection{Estimation of the compression modulus}

The value of the compression modulus $k_{\mathrm{m}}$ used in the simulation is based on the experimentally determined average contact time of microtubules with the cell poles before they undergo a catastrophe, $90 \mathrm{~s}$ measured in wild-type fission yeast cells [6]. Based on the force production model described above, the instantaneous catastrophe rate as a function of the compression, $l-L$, equals:

$$
r_{\mathrm{c}}(l-L)=r_{\mathrm{c}}^{(0)} e^{k_{\mathrm{m}}(l-L) / F_{\mathrm{s}}}=r_{\mathrm{c}}^{(0)} e^{\kappa(l-L)}
$$

where we defined $\kappa=k_{\mathrm{m}} / F_{\mathrm{S}}$ as a compressibility factor that has a dimension of reciprocal length. Now consider the survival probability, $S(t \mid L)$, of a growing microtubule, which first contacts the barrier at $t=0$ with length $L$ and then increases its compression, while the distance between nucleation point and barrier remains constant. This quantity obeys the equation

$$
\frac{\mathrm{d}}{\mathrm{d} t} S(t \mid L)=-r_{\mathrm{c}}(t) S(t \mid L), \quad S(0 \mid L)=1
$$

where $r_{\mathrm{c}}(t)$ is the instantaneous catastrophe rate, which increases as the load increases. To determine the latter, we first solve for the stored length at time $t$, assuming no catastrophe has yet occurred

$$
\frac{\mathrm{d}}{\mathrm{d} t} l(t)=v_{\mathrm{g}}\left(F(l(t)-L)=v_{\mathrm{g}}^{(0)} e^{-\kappa(l(t)-L)}\right.
$$


which is readily solved to yield

$$
l(t)=L+\frac{1}{\kappa} \ln \left(1+\kappa v_{\mathrm{g}}^{(0)} t\right)
$$

Inserting into Eq. 16 yields

$$
r_{\mathrm{c}}(t)=r_{\mathrm{c}}^{(0)}\left(1+\kappa v_{\mathrm{g}}^{(0)} t\right)
$$

i.e. the catastrophe rate increases linearly in time. This allows us to integrate Eq. 14 to obtain

$$
S(t \mid L)=e^{-r_{\mathrm{c}}^{(0)} t\left(1+\kappa v_{\mathrm{g}}^{(0)} t / 2\right)}
$$

The waiting time distribution can be calculated from Eq. 17 and is defined as:

$$
w(t)=-\frac{\mathrm{d} S}{\mathrm{~d} t}(t)=r_{\mathrm{c}}(t) S(t)
$$

From the survival probability, we can determine the mean time to catastrophe $\tau_{\mathrm{c}}$ as

$$
\left\langle\tau_{\mathrm{c}}\right\rangle=\int_{0}^{\infty} \mathrm{d} t t w(t)=\int_{0}^{\infty} \mathrm{d} t S(t \mid L)
$$

where the second identity follows from integration by parts. By insertion of Eq. 21, we now obtain:

$$
\left\langle\tau_{\mathrm{c}}\right\rangle=\frac{1}{r_{\mathrm{c}}^{(0)}} \sqrt{\pi \alpha} e^{\alpha} \operatorname{Erfc}(\sqrt{\alpha})
$$

where $\alpha=r_{\mathrm{c}}^{(0)} / 2 \kappa v_{\mathrm{g}}^{(0)}$ and Erfc is the complementary error function

$$
\operatorname{Erfc}(z)=\frac{2}{\sqrt{\pi}} \int_{z}^{\infty} e^{-t^{2}}
$$

Equation 24 allows $\kappa$ to be extracted by numerical inversion from the mean experimental waiting time of $90 \mathrm{~s}$. The so obtained value of $\sim 0.5 \mu \mathrm{m}^{-1}$ (see Table 2) indicates that the typical forces involved are around $1 \mathrm{pN}$. At $1 \mathrm{pN}$ compression force, a microtubule with a persistence length of $5 \mathrm{~mm}$ that is unconstrained on both sides, will buckle only if it is 14 $\mu \mathrm{m}$ or longer [42]. This a posteriori validates that we can neglect microtubule buckling in our model, certainly at the length scale of the internuclear distance. To further validate our approach, we implemented confined growth, stored length and compression into a simulation of a mononucleated cell and analysed the waiting time of microtubules before undergoing a catastrophe. The mean waiting time for the simulation $(88.9 \pm 0.1 \mathrm{~s}(\mathrm{SE}))$ was essentially equal to the experimental waiting time, demonstrating that the force production model was correctly implemented in the simulation.

\subsection{Nucleation rate}

The remaining parameter to be determined is the rate of microtubule nucleation per nucleus. Microtubule nucleation in fission yeast not only occurs at spindle pole bodies (SPBs), but also along existing microtubules and on the nuclear envelope [16]. Together these processes generate microtubule growth that is directed away from nuclei. The total rate of microtubule nucleation in cells is difficult to estimate experimentally because of the multiple nucleation processes involved. To capture the essence of the mechanism in our simulation, we nucleated individual microtubules from nucleation sites on the nuclei. With equal probability, microtubules were nucleated either on the left- or the right edge of nuclei and then grew away 
from them in that direction. It has been shown that on average 1.5 microtubules are in contact with the cell tips in wild-type fission yeast cells with a single nucleus [37]. Therefore, we varied the nucleation rate in a simulation of a mononucleated cells to achieve the observed number of microtubule-wall interactions. This rate, $r_{n}=0.0275 \mathrm{~s}^{-1}$ nucleus ${ }^{-1}$ (Table 2), was then used in simulations of tetranucleated cells. The average number of microtubules per nucleus for this nucleation rate, including those that do not generate forces, was between 5 and 6 (Fig. 4). This compares well to the situation in wild-type cells, where each nucleus has between three to five IMAs, each consisting out of about three microtubules [16]. In our 1-dimensional simulation, we neglect the assembly of microtubules into IMAs; instead, we have individual microtubules, representing approximately the same number of microtubules that are mechanically coupled by their individual attachments to the nucleus.

\subsection{Validation of the simulation}

To test whether the modelled forces and microtubule dynamics yield nuclear motion that is comparable to previous work, we determined the distribution of nuclear midpoint positions in simulated mononucleated cells. The standard deviation of the distribution $(0.88 \mu \mathrm{m})$ was larger but nevertheless similar to values obtained in more complex 3D simulations with equal parameter values for microtubule dynamics and cytoplasmic viscosity (values up to $0.5 \mu \mathrm{m}$ ) [36]. Note that by the increasing nuclear drag in the simulation, we could further narrow the distribution, but we chose to keep the values as they are since they are based on the experimentally determined diameter of cells and nuclei.

Supplementary Information The online version contains supplementary material available at https://doi. org/10.1140/epjp/s13360-021-01756-7.

Acknowledgements We thank F. Chang, K. Gould, M. Sato, P.T. Tran and YGRC/NBRP, Japan for strains; D. McCollum, S. Trautmann, A. Gladfelter, A. Akhmanova, M. Dogterom and T. Ketelaar for discussions; K. Gökhan Süslü and H. Kieft for assistance in the lab; and J. de Keijzer for help with MatLab. BM, JT and MJ acknowledge support from the Foundation for Fundamental Research on Matter (FOM) and the Division for Earth and Life Sciences (ALW), formerly part of the Dutch Research Council (NWO). The work of BM is part of the Dutch Research Council (NWO) and was performed at the research institute AMOLF.

Data Availability Statement This manuscript has associated data in a data repository. [Authors' comment: The simulation code is publicly available on: https://github.com/BelaMulder/nucleosim.]

Open Access This article is licensed under a Creative Commons Attribution 4.0 International License, which permits use, sharing, adaptation, distribution and reproduction in any medium or format, as long as you give appropriate credit to the original author(s) and the source, provide a link to the Creative Commons licence, and indicate if changes were made. The images or other third party material in this article are included in the article's Creative Commons licence, unless indicated otherwise in a credit line to the material. If material is not included in the article's Creative Commons licence and your intended use is not permitted by statutory regulation or exceeds the permitted use, you will need to obtain permission directly from the copyright holder. To view a copy of this licence, visit http://creativecommons.org/licenses/by/4.0/.

\section{References}

1. I.H. Jain, V. Vijayan, E.K. O'Shea, Spatial ordering of chromosomes enhances the fidelity of chromosome partitioning in cyanobacteria. Proc. Natl. Acad. Sci. U. S. A. 109, 13638-43 (2012)

2. D.F. Savage, B. Afonso, A.H. Chen, P.A. Silver, Spatially ordered dynamics of the bacterial carbon fixation machinery. Science 327, 1258-61 (2010)

3. W.F. Marshall, C. Kintner, Cilia orientation and the fluid mechanics of development. Curr. Opin. Cell Biol. 20, 48-52 (2008) 
4. V.E. Foe, C.M. Field, G.M. Odell, Microtubules and mitotic cycle phase modulate spatiotemporal distributions of F-actin and myosin II in Drosophila syncytial blastoderm embryos. Development 127, 1767-87 (2000)

5. T. Metzger, V. Gache, M. Xu, B. Cadot, E.S. Folker, B.E. Richardson, E.R. Gomes, M.K. Baylies, MAP and kinesin-dependent nuclear positioning is required for skeletal muscle function. Nature 484, 120-4 (2012)

6. P.T. Tran, L. Marsh, V. Doye, S. Inoué, F. Chang, A mechanism for nuclear positioning in fission yeast based on microtubule pushing. J. Cell Biol. 153, 397-411 (2001)

7. K. Scheffler, R. Minnes, V. Fraisier, A. Paoletti, P.T. Tran, Microtubule minus end motors kinesin-14 and dynein drive nuclear congression in parallel pathways. J. Cell Biol. 209, 47-58 (2015)

8. C.L. Troxell, M.A. Sweezy, R.R. West, K.D. Reed, B.D. Carson, A.L. Pidoux, W.Z. Cande, J.R. McIntosh, pk11(+)and klp2(+): two kinesins of the Kar3 subfamily in fission yeast perform different functions in both mitosis and meiosis. Mol. Biol. Cell. 12, 3476-88 (2001)

9. S. Mana-Capelli, J.R. McLean, C.-T. Chen, K.L. Gould, D. McCollum, The kinesin-14 Klp2 is negatively regulated by the SIN for proper spindle elongation and telophase nuclear positioning. Mol. Biol. Cell. 23, 4592-600 (2012)

10. P. Nurse, P. Thuriaux, K. Nasmyth, Genetic control of the cell division cycle in the fission yeast Schizosaccharomyces pombe. Mol. Gen. Genet. 146, 167-78 (1976)

11. R.E. Carazo-Salas, C. Antony, P. Nurse, The kinesin Klp2 mediates polarization of interphase microtubules in fission yeast. Science 309, 297-300 (2005)

12. M.E. Janson, R. Loughlin, I. Loïodice, C. Fu, D. Brunner, F.J. Nédélec, P.T. Tran, Crosslinkers and motors organize dynamic microtubules to form stable bipolar arrays in fission yeast. Cell 128, 357-68 (2007)

13. E.H. Lieb, D.C. Mattis, Mathematical Physics in One Dimension: Exactly Soluble Models of Interacting Particles (Academic Press, London, 1966)

14. R.R. Daga, F. Chang, Dynamic positioning of the fission yeast cell division plane. Proc. Natl. Acad. Sci. U. S. A. 102, 8228-32 (2005)

15. D.R. Drummond, R.A. Cross, Dynamics of interphase microtubules in Schizosaccharomyces pombe. Curr. Biol. 10, 766-75 (2000)

16. M.E. Janson, T.G. Setty, A. Paoletti, P.T. Tran, Efficient formation of bipolar microtubule bundles requires microtubule-bound gamma-tubulin complexes. J. Cell Biol. 169, 297-308 (2005). https://doi.org/10.108 3/jcb.200410119

17. J. Howard, Elastic and damping forces generated by confined arrays of dynamic microtubules. Phys. Biol. 3, 54-66 (2006). https://doi.org/10.1088/1478-3975/3/1/006

18. M. Dogterom, B. Yurke, Microtubule dynamics and the positioning of microtubule organizing centers. Phys. Rev. Lett. 81, 485-488 (1998)

19. J.C. Bruusgaard, K. Liest $\varnothing 1$, M. Ekmark, K. Kollstad, K. Gundersen, Number and spatial distribution of nuclei in the muscle fibres of normal mice studied in vivo. J. Physiol. 551, 467-478 (2003). https://doi. org/10.1113/jphysiol.2003.045328

20. P. Gimpel, Y.L. Lee, R.M. Sobota, A. Calvi, V. Koullourou, R. Patel, K. Mamchaoui, F. Nédélec, S. Shackleton, J. Schmoranzer, B. Burke, B. Cadot, E.R. Gomes, Nesprin-1 $\alpha$-dependent microtubule nucleation from the nuclear envelope via Akap450 is necessary for nuclear positioning in muscle cells. Curr. Biol. 27, 2999-3009.e9 (2017). https://doi.org/10.1016/j.cub.2017.08.031

21. A. Manhart, S. Windner, M. Baylies, A. Mogilner, Mechanical positioning of multiple nuclei in muscle cells. PLoS Comput. Biol. 14, 1-25 (2018). https://doi.org/10.1371/journal.pcbi.1006208

22. C.A. Anderson, U. Eser, T. Korndorf, M.E. Borsuk, J.M. Skotheim, A.S. Gladfelter, Nuclear repulsion enables division autonomy in a single cytoplasm. Curr. Biol. 23, 1999-2010 (2013)

23. J. Baker, W.E. Theurkauf, G. Schubiger, Dynamic changes in microtubule configuration correlate with nuclear migration in the preblastoderm Drosophila embryo. J. Cell Biol. 122, 113-21 (1993)

24. R.C. Brown, B.E. Lemmon, The cytoskeleton and spatial control of cytokinesis in the plant life cycle. Protoplasma 215, 35-49 (2001)

25. E. McNaughton, L. Goff, The role of microtubules in establishing nuclear spatial patterns in multinucleate green algae. Protoplasma 157, 19-37 (1990). https://doi.org/10.1007/BF01322636

26. R. Gibeaux, A.Z. Politi, P. Philippsen, F. Nédélec, Mechanism of nuclear movements in a multinucleated cell. Mol. Biol. Cell. 28, 645-660 (2017). https://doi.org/10.1091/mbc.E16-11-0806

27. S. Grava, M. Keller, S. Voegeli, S. Seger, C. Lang, P. Philippsen, Clustering of nuclei in multinucleated hyphae is prevented by dynein-driven bidirectional nuclear movements and microtubule growth control in Ashbya gossypii. Eukaryot. Cell. 10, 902-15 (2011)

28. R. Gibeaux, C. Lang, A.Z. Politi, S.L. Jaspersen, P. Philippsen, C. Antony, Electron tomography of the microtubule cytoskeleton in multinucleated hyphae of Ashbya gossypii. J. Cell Sci. 125, 5830-9 (2012) 
29. M.H. Wilson, E.L.F. Holzbaur, Opposing microtubule motors drive robust nuclear dynamics in developing muscle cells. J. Cell Sci. 125, 4158-69 (2012)

30. T. Sugawara, K. Kaneko, Chemophoresis as a driving force for intracellular organization: theory and application to plasmid partitioning. Biophysics (Oxford) 7, 77-88 (2011). https://doi.org/10.2142/biophysics. 7.77

31. A.G. Vecchiarelli, K.C. Neuman, K. Mizuuchi, A propagating ATPase gradient drives transport of surfaceconfined cellular cargo. Proc. Natl. Acad. Sci. U. S. A. 111, 4880-5 (2014)

32. K.A. Bohnert, J.-S. Chen, D.M. Clifford, C.W. Vander Kooi, K.L. Gould, A link between aurora kinase and $\mathrm{Clp} 1 / \mathrm{Cdc} 14$ regulation uncovered by the identification of a fission yeast borealin-like protein. Mol. Biol. Cell. 20, 3646-59 (2009)

33. S.V. Bratman, F. Chang, Stabilization of overlapping microtubules by fission yeast CLASP. Dev. Cell. 13, 812-27 (2007). https://doi.org/10.1016/j.devcel.2007.10.015

34. P.T. Tran, A. Paoletti, F. Chang, Imaging green fluorescent protein fusions in living fission yeast cells. Methods 33, 220-5 (2004)

35. Z.W. Salsburg, R.W. Zwanzig, J.G. Kirkwood, Molecular distribution functions in a one-dimensional fluid. J. Chem. Phys. 21, 1098 (1953)

36. D. Foethke, T. Makushok, D. Brunner, F. Nédélec, Force- and length-dependent catastrophe activities explain interphase microtubule organization in fission yeast. Mol. Syst. Biol. 5, 241 (2009). https://doi. org/10.1038/msb.2008.76

37. R.R. Daga, A. Yonetani, F. Chang, Asymmetric microtubule pushing forces in nuclear centering. Curr. Biol. 16, 1544-50 (2006). https://doi.org/10.1016/j.cub.2006.06.026

38. F.R. Neumann, P. Nurse, Nuclear size control in fission yeast. J. Cell Biol. 179, 593-600 (2007). https:// doi.org/10.1083/jcb.200708054

39. M. Dogterom, B. Yurke, Measurement of the force-velocity relation for growing microtubules. Science 278, 856-60 (1997)

40. M. Janson, M. Dogterom, Scaling of microtubule force-velocity curves obtained at different tubulin concentrations. Phys. Rev. Lett. 92, 1-4 (2004). https://doi.org/10.1103/PhysRevLett.92.248101

41. M.E. Janson, M.E. de Dood, M. Dogterom, Dynamic instability of microtubules is regulated by force. J. Cell Biol. 161, 1029-34 (2003)

42. L.D. Landau, L.P. Pitaevskii, A.M. Kosevich, E.M. Lifshitz, Theory of Elasticity, 3rd edn. (ButterworthHeinemann, 1986)

43. M. Sato, N. Okado, Y. Kakui, M. Yamamoto, M. Yoshida, T. Toda, Nucleocytoplasmic transport of Alp7/TACC organizes spatiotemporal microtubule formation in fission yeast. EMBO J. 10, 1161-1167 (2009) 